\title{
DISCRIMINACIÓN DE PRECIOS DE SEGUNDO GRADO: EL CASO DE TARIFAS MULTI-CLASE PARA EL REGISTRO MARCARIO EN COLOMBIA*
}

\author{
JUAN PABLO HERRERA SAAVEDRA**, NATALIA CANTOR VARGAS $S^{* * * *}$, \\ JENNY-PAOLA LIS-GUTIÉRREZ $Z^{* * * * *} \&$ JACOBO ALBERTO CAMPO ROBLEDO****** \\ SUPERINTENDENCIA DE INDUSTRIA Y COMERCIO (COLOMBIA)
}

Recibido/ Received/ Recebido: 20/04/2013 - Aceptado/ Accepted / Aprovado: 19/03/2014

\begin{abstract}
Resumen
Este documento presenta una aplicación de discriminación de segundo grado a las tarifas de propiedad industrial, en particular al sistema multi-clase, esto a propósito de la adhesión de Colombia al Protocolo de Madrid en 2011. La metodología consistió en la construcción de un modelo para establecer una estructura tarifaria óptima de registro de marcas con dos objetivos: (i) cumplir con las obligaciones dispuestas en el TLT e incentivar la utilización del sistema multi-clase, y (ii) identificar un mecanismo que minimizara los efectos negativos, que en materia de recaudo podría enfrentar la autoridad de propiedad industrial, con la entrada en vigencia de dicho acuerdo. Dentro de los principales hallazgos del estudio se identificó la no existencia inequívoca inversa entre tarifa marcaria y número de solicitudes, lo que da espacio a considerar múltiples factores que pueden incidir en la demanda del servicio de registros marcarios distintos a su precio. Igualmente, se estableció que este sistema de tarifas diferenciadas amortiguó el descenso de los ingresos de la autoridad administradora de propiedad industrial en Colombia dadas las características de la demanda del servicio observado. Palabras clave: Tratado sobre derecho de marcas (TLT), Discriminación de segundo grado, Propiedad industrial, Protocolo de Madrid, Marcas.
\end{abstract}

\section{SECOND LEVEL PRICE DIFFERENTIATION: CASE OF MULTICLASS PRICE FOR TRADEMARK REGISTRY IN COLOMBIA}

\begin{abstract}
This paper shows the implementation of a second level price differentiation of industrial property price, in particular the multi-class system, this is because Colombia's accession to Madrid's Protocol in 2011. The methodology is based on a model to establish an optimal trademark price structure
\end{abstract}

* Investigación elaborada por el grupo de Estudios Económicos de la Superintendencia de Industria y Comercio de Colombia.

* Magister en Ciencias Económicas. Coordinador del Grupo de Estudios Económicos y Asesor del Superintendente de Industria y Comercio. Profesor de las universidades Nacional y Externado de Colombia. Correo electrónico: estudioseconomicos@sic.gov.co

*** Economista y estudiante de maestría en Economía. Su participación en la elaboración de este documento se realizó entre abril y agosto de 2012, momento en el cual estaba vinculada como economista al Grupo de Estudios Económicos de la Superintendencia de Industria y Comercio. Correo electrónico: natalia.cantor@urosario.edu.co

***** Estudiante de Doctorado en Industria y Organizaciones. Magister en Análisis de Problemas Políticos, Económicos e Internacionales; Magister en Sociedades Contemporáneas Comparadas; Especialidad en Geografía y Planeación. Economista del Grupo de Estudios Económicos de la Superintendencia de Industria y Comercio. Correo electrónico: jgutierrez@sic.gov.co

****** Magister en Economía. Economista del Grupo de Estudios Económicos de la Superintendencia de Industria y Comercio. Correo electrónico: jcampo@sic.gov.co 
with two objectives: (i) to accomplish commitments under the TLT and encourage the use of multiclass system, and (ii) to identify a mechanism to reduce negative effects, which in terms of collection could face industrial property authority, based on the agreement in force. Among main findings, the inverse unequivocal existence between price and number of requests was identified, considering multiple factors that can affect the demand trademark service. Additionally, it was established that this differentiated price system leveraged the decrease on revenues from the industrial property management authority in Colombia given the observed demand service characteristics.

Keywords: Trademark Law Treaty (TLT), Second level differentiation, Industrial property, Madrid Protocol, Trademarks.

\title{
DISCRIMINAÇÃO DE PREÇOS DE SEGUNDO GRAU: O CASO DAS TARIFAS MULTICLASSE PARA O REGISTO DE MARCAS NA COLÔMBIA
}

\section{Resumo}

\begin{abstract}
Este documento apresenta uma aplicação de discriminação de segundo grau às tarifas de propriedade industrial, em particular ao sistema multiclasse, isto a propósito da adesão da Colômbia ao Protocolo de Madri em 2011. A metodologia consistiu na construção de um modelo para estabelecer uma estrutura tarifária ótima de registo de marcas com dois objetivos: (i) cumprir com as obrigações dispostas no TLT e incentivar a utilização do sistema multiclasse, e (ii) identificar um mecanismo que minimizasse os efeitos negativos que poderia enfrentar a autoridade de propriedade industrial em matéria de arrecadação, com a entrada em vigência de dito acordo. Dentro dos principais resultados do estudo se identificou a não existência inequívoca inversa entre tarifa de marca e número de solicitações, o que dá espaço a considerar múltiplos fatores que podem incidir na demanda do serviço de registos de marcas diferentes a seu preço. Igualmente, se estabeleceu que este sistema de tarifas diferenciadas amorteceu o descenso dos rendimentos da autoridade administradora de propriedade industrial na Colômbia dadas as características da demanda do serviço observado.
\end{abstract}

Palavras chave: Tratado sobre direito de marcas (TLT), Discriminação de segundo grau, Propriedade industrial, Protocolo de Madri, Marcas.

Herrera, J., Cantor, N., Lis-Gutierrez, J. \& Campo, J. (2014) Discriminación de precios de segundo grado: el caso de tarifas multi-clase para el registro marcario en Colombia (2012). En: Revista de la Facultad de Ciencias Económicas de la Universidad Militar Nueva Granada. rev.fac.cienc.econ, XXII (1)

JEL: D04, D40, L51.

\section{Introducción}

El Tratado sobre derecho de Marcas (TLT, Trademark Law Treaty) adoptado en Ginebra el 27 de octubre de 1994, tiene como objetivo principal la armonización y simplificación de los procedimientos para registro y demás trámites de marcas, ante las oficinas de registro de los países que hacen parte del tratado. Lo anterior con el fin de reducir los costos de los trámites, y uniformizar y agilizar los procedimientos nacionales y regionales de registro de marcas. El TLT consta de 25 artículos, su adhesión por parte de Colombia se llevó a cabo el día 13 de enero de 2012 y su entrada en vigor empezó el día 13 de abril de 2012. En 1996 había 6 partes (países) contratantes del tratado, cifra que aumentó a 26 en 2000 y a 50 en 2012.

En ese contexto, el presente documento tiene el propósito de proponer un mecanismo de definición de 
tarifas marcarias multi-clase basado en la idea de discriminación de segundo grado, que pueda ser replicado para otras tarifas de propiedad industrial $\mathrm{u}$ otras instituciones responsables de trámites gubernamentales. Para el efecto, fue construido un modelo que permitiera establecer una estructura tarifaria óptima de registro de marcas con dos propósitos: (i) cumplir con las obligaciones dispuestas en el TLT e incentivar la utilización del sistema multi-clase, (ii) identificar un mecanismo que minimizara los efectos negativos, que en materia de recaudo podría enfrentar la autoridad de propiedad industrial, con la entrada en vigencia de dicho acuerdo.

Para contextualizar al lector sobre el problema, el documento presenta una breve revisión de la literatura. La siguiente sección presenta algunos hechos estilizados relacionados con el tema. El tercer apartado propone un modelo de fijación tarifaria de solicitud de registro de marcas que se implementó para reducir el riesgo de caída en el recaudo. En el cuarto segmento se presenta un ejercicio de impacto sobre los ingresos de la Entidad y de la propuesta tarifaria que se aplicó para el registro de las afectaciones o modificaciones ${ }^{1}$. Finalmente, se formulan las conclusiones y recomendaciones derivadas del ejercicio.

\section{Revisión de la Literatura}

En primera instancia es de mencionar el trabajo de Corcione (2010) en el que se realiza un análisis descriptivo sobre el TLT y los retos para la Superintendencia de Industria y Comercio como oficina nacional de propiedad industrial frente a la entrada en vigencia del tratado. El contexto presentado en el referido trabajo motiva una redefinición del sistema tarifario vigente hasta el año 2011 en Colombia. Para la identificación de una propuesta alterna, se avanzó en una revisión de algunos documentos relacionados con la teoría de discriminación de segundo grado que se presenta a continuación.

La literatura internacional sobre estructuras tarifarias en dos partes es amplia y diversa, sin embargo, los temas aplicados a tarifas de propiedad industrial no resultan igual de abundantes, de allí la relevancia de este documento. Los trabajos más recientes se concentran en cuatro temáticas: (i) la coordinación entre las firmas y el análisis de canales de mercadeo (Lambertini, 2013; Ma, Wang \& Shang, 2013; Goering, 2012; Matsui, 2012); (ii) aplicación de la tarifa en dos partes a los sistemas de generación de energía eléctrica -para el caso brasilero se encuentra el trabajo de Steele Santos, Coradi Leme \& Galvão (2012), el caso español lo abordan Oliveira, Ruiz \& Conejo, 2013 y la generación eléctrica en Perú es estudiada por Ruiz \& Rosellón (2012)-; (iii) aplicación a modelos de competencia imperfecta -Willington \& Li Ning (2014) al monopolio y Ferreira \& Bode (2013) y Chen, Zhang \& Sun (2012) al modelo de Stackelberg \& Cournot-, (iv) y licenciamiento de tecnología e innovación (Mukherjee \& Mukherjee, 2013; Chang, Hwang \& Peng, 2013; Kim, Park \& Lee (2012).

En años anteriores, Li \& Yanagawa (2011) expusieron una aplicación de la tarifa de dos partes en la licencia de patentes, considerando un modelo de duopolio de Cournot-Stackelberg con productos diferenciados. Contrastaron sus resultados con los obtenidos por Wang (2002), quien estudió las licencias por medio de una cuota fija y la concesión de licencias a través de un duopolio de Cournot, encontrando que las licencias de cuota fija son siempre preferidas por los consumidores, incluso frente a las licencias libres.

Mendi, Moner-Colonques \& Sempere-Monerris (2011) propusieron un modelo que incluía la discriminación de segundo grado en contratos de transferencia de tecnología entre empresas matrices filiales y rivales, y analizaron el efecto del mecanismo de fijación de precios sobre la competencia. Este trabajo, proporcionó adicionalmente una visión cuantitativa del mercado internacional de tecnología.

Por su parte, Cornia, Gerardi \& Shapiro (2011) identificaron que el ciclo económico afecta el grado en el cual las firmas emplean la discriminación entre los diferentes tipos de consumidores, por medio de

Trámites que se presentan una vez se ha concedido el registro de marca. 
un modelo teórico aplicado al mercado de las aerolíneas entre el primer trimestre de 1993 y el cuarto trimestre de 2009.

Por su parte, Rosellon, Tregear \& Zenon (2012) modelaron el rebalanceo de una tarifa en dos partes para el mercado eléctrico mayorista de Ontario en Canadá. Concluyeron que los precios convergen al costo marginal de generación, la renta de congestión disminuye y el bienestar social se incrementa. Bonnet \& Dubois (2010) presentaron un modelo de contrato vertical para estimar la demanda y la oferta estructurales, permitiendo un esquema de tarifas en dos partes, con el fin de analizar el caso del agua embotellada en Francia, en los contratos entre productores y comerciantes al detal.

Desde otra óptica, Gotlibovski \& Kahana (2009) compararon Ilustración y matemáticamente dos tipos de discriminación de segundo grado (tarifa en dos partes y paquetes precio-cantidad), considerando dos clases de consumidores. Inderst \& Shaffer (2009) elaboraron un modelo en el cual la tarifa en dos partes era aplicada a los contratos celebrados entre el monopolista y los proveedores.

Trabajos anteriores analizaron diferentes mercados alrededor de todo el mundo mediante el estudio de la discriminación de segundo grado. Por ejemplo, Braverman, Guasch \& Salop (1983) estudiaron la compra de garantías para los productos adquiridos, como una forma de discriminación de precios de segundo grado aplicado al caso de Disneylandia, solucionando analíticamente el modelo de monopolio. Wakita (2001) presentó una aplicación de la tarifa en dos partes a la determinación de salarios en Japón. Yao (2002) estudió el uso de la discriminación de segundo grado para modelar la corrupción en China \& Felder (2004) elaborando una aplicación de la discriminación de precios de segundo grado a la regulación del precio del mercado de medicamentos.

\section{Hechos estilizados}

A efectos de contextualizar al lector, resulta importante señalar algunos datos que ilustran la situación relativa de Colombia frente a otros países en lo relacionado con el valor de registro marcario vigente a diciembre de 2012. El ejercicio realizado buscó contrastar la situación colombiana frente a 49 países, encontrando algunos elementos llamativos que son enunciados a continuación.

Al considerar el valor de registro de una marca $^{2}$ con afectación a una sola clase ${ }^{3}$, Colombia se encontraba en la décima posición entre los 50 países analizados siendo la primera posición la más costosa- (Ilustración A1 en el anexo); para la afectación a dos clases Colombia ascendía a la quinta posición (Ilustración A2 en el anexo) y para la afectación a tres clases Colombia a la cuarta posición (Ilustración A3 en el anexo).

Cuba, Japón, Perú, Ghana, Suiza y Ucrania aplicaban una tarifa en dos partes.

Noruega, Finlandia, Benelux, Bonaire, San Marino, Bulgaria, Unión Europea, Curazao y Dinamarca manejaban una tarifa multi-clase (entre una y tres) para marcas colectivas y no colectivas.

2 De acuerdo con la SIC $(2008$, 13), la marca es: un signo distintivo que sirve para identificar productos o servicios en el mercado. Es el signo que otorgará a una persona y/o empresa la capacidad de identificar sus productos o servicios de otros idénticos o similares existentes en el mercado.

Las marcas pueden consistir en palabras, letras, números, dibujos, imágenes, formas, colores, logotipos, figuras, símbolos, gráficos, monogramas, retratos, etiquetas, emblemas, escudos, sonidos, o combinación de estos elementos".

3 De acuerdo con la SIC $(2008,15)$, una clase es: es un término utilizado para referirse a ese grupo de productos o servicios que guardan relación entre sí, agrupados de acuerdo con una clasificación aceptada internacionalmente denominada "Clasificación de Niza". La clasificación internacional está compuesta por unos títulos de las clases y unas notas explicativas que, a su vez, incluyen exclusiones, y está complementada por un listado alfabético por producto o servicio. De manera general, los productos se clasifican así:

- productos acabados por su función.

- materias primas en atención a su materia.

- productos accesorios no separables, van con el principal.

- estuches con el producto que van a contener. 
Belarus, contaba con una tarifa para registro entre una y tres clases para marcas no colectivas.

Estonia era el único país con una tarifa para registro entre una y tres clases para marcas colectivas y que no lo hacía para marcas no colectivas.

Dado que varios países hacen una distinción entre marca no colectiva y colectiva, en la Ilustración A4 del Anexo se presenta la diferencia porcentual para cada uno de los países. Exceptuando Noruega e Islandia, en el resto de casos la diferencia era estrictamente positiva, siendo la más alta $401 \%$ en Grecia y la más pequeña $8 \%$ (Bahréin).

$\mathrm{El}$ incremento porcentual para registro marcario, se consolida en el Tabla 1. La mayor parte de los países aplicaba un aumento entre el 75 y el $100 \%$, al comparar la afectación entre 1 y 2 clases. Entre 1 y 3 clases el incremento se concentraba en el rango superior a $100 \%$. Entre 2 y 3 clases los países se ubicaban, principalmente, en las categorías entre $0 \%$ y $25 \%$, y $50 \%$ y $75 \%$.

Al comparar el registro de marcas en 2011 (Tabla 2) puede verse que países con menores tarifas que Colombia presentaron un registro más bajo de marcas -como Venezuela y Uruguay, en la región-; y países con tarifas más altas tuvieron un mejor comportamiento, como es el caso de Ucrania y Australia. Algo similar a lo anterior se identifica al analizar las solicitudes de registro de marcas para 2011; Venezuela y Cuba a pesar de tener menores tarifas que Colombia presentan solicitudes de marcas más reducidas, mientras que en Japón y Australia, cuyas oficinas de registro aplican tarifas más altas, presentan un mejor comportamiento en las solicitudes (Tabla 3). Lo anterior evidencia la existencia de factores diferentes a la tarifa como determinantes de la actividad marcaria.

\section{Modelo de determinación tarifaria sistema multi-clase}

Dentro de la simplificación y la armonización de los procedimientos y la eliminación de obstáculos, el TLT dispone que las oficinas de marcas establezcan un sistema de registro multi-clase, esto es, permite que una solicitud única de registro de marca pueda cubrir varias clases de productos o servicios. Frente a este asunto, el Artículo 6 del TLT establece que "cuando se hayan incluido en una solicitud única productos y/o servicios que pertenezcan a varias clases de la Clasificación de Niza, dicha solicitud dará por resultado un registro único" (OMPI, 1994, artículo 6).

Frente a lo anterior, fue necesario sugerir ajustes al sistema de registro de marcario (en la que se hacía una solicitud de registro de marca por cada clase a registrar) $e$ implementar las medidas necesarias para que esta disposición del TLT no afectara las finanzas de la Entidad.

\subsection{Modelo teórico}

El modelo está inspirado en la teoría de Discriminación de precios de segundo grado ${ }^{12}$, la cual establece el cobro de diferentes precios dependiendo de la cantidad o "bloque" de bienes consumidos.

Utilizando esta idea, se propuso la implementación de una estructura tarifaria que se asemejara a una tabla de precios, en la cual se aplicara un descuento por cantidad, es decir, que establezca el cobro de tarifas medias decrecientes a medida que se incrementa el número de clases a registrar en una solicitud única de registro de marca. Frente a la política de tarifa única, esta nueva estructura tarifaria ofrece mayores beneficios a los empresarios que registran marcas clasificadas en múltiples clases y amortigua el descenso de los ingresos de la Entidad al hacer un único registro de marca con afectación multi-clase.

En este contexto, se establece la tarifa lineal que se cobraría a cada clases de productos o servicios registrados en una solicitud única de registro de marca. Dicha tarifa cuenta con dos partes y se expresa de la siguiente forma:

$$
I_{n}=n \cdot I-s \sum_{i=0}^{n-1} i
$$

En donde:

$I_{n}$ es la tarifa total que se cobra al registrarclases en una solicitud única de registro de marca. 
Tabla 1. Incremento porcentual para registro marcario con afectación entre 1 y 2 clases, 1 y 3 clases, y 2 y 3 clases ${ }^{4}$

\begin{tabular}{|c|c|c|c|c|c|c|}
\hline Descripción & $\begin{array}{l}\text { Sin incremento por } \\
\text { aplicar tarifa única } \\
\text { con afectación a } 1 \\
\text { o a } 3 \text { clases }\end{array}$ & $\begin{array}{c}\text { Incremento [0\% } \\
-25 \%]\end{array}$ & $\begin{array}{l}\text { Incremento } \\
(25 \%-50 \%]\end{array}$ & $\begin{array}{l}\text { Incremento entre } \\
(50 \%-75 \%]\end{array}$ & $\begin{array}{l}\text { Incremento entre } \\
(75 \%-100 \%]\end{array}$ & Superior al $100 \%$ \\
\hline $\begin{array}{l}\text { Incremento porcen- } \\
\text { tual para registro } \\
\text { marcario con } \\
\text { afectación entre } 1 \text { y } \\
2 \text { clases }\end{array}$ & $\begin{array}{c}\text { Estonia, Belarus, } \\
\text { Noruega, Finlandia, } \\
\text { Benelux, Bonaire, } \\
\text { San Marino, Bul- } \\
\text { garia, } \\
\text { Unión Europea, } \\
\text { Curazao, Dinamarca }\end{array}$ & $\begin{array}{l}\text { Tayikistán, Armenia, } \\
\text { Uzbequistán, Suiza, } \\
\text { Japón, Turquía, } \\
\text { Ucrania, República } \\
\text { de Moldova, Is- } \\
\text { landia }\end{array}$ & $\begin{array}{l}\text { Grecia, Cuba, } \\
\text { Reino Unido, } \\
\text { Irlanda, Italia, } \\
\text { Georgia, Kyr- } \\
\text { gyzstan, Suecia }\end{array}$ & $\begin{array}{c}\text { China, Turkmenis- } \\
\text { tán, Ghana }\end{array}$ & $\begin{array}{l}\text { Israel, Vietnam, Perú, } \\
\text { Colombia, México, } \\
\text { Bahréin, Omán, } \\
\text { Australia, Chile, Costa } \\
\text { Rica, Estados Unidos, } \\
\text { Filipinas, Nueva } \\
\text { Zelandia, República de } \\
\text { Corea, Singapur, Siria, } \\
\text { Venezuela }\end{array}$ & \\
\hline $\begin{array}{l}\text { Incremento porcen- } \\
\text { tual para registro } \\
\text { marcario con } \\
\text { afectación entre } 1 \text { y } \\
3 \text { clases }\end{array}$ & $\begin{array}{l}\text { Estonia, Belarus, } \\
\text { Noruega, Finlandia, } \\
\text { Benelux, Bonaire, } \\
\text { San Marino, Bulga- } \\
\text { ria, Unión Europea, } \\
\text { Curazao, Dinamarca }\end{array}$ & $\begin{array}{l}\text { Tayikistán, Armenia, } \\
\text { Uzbequistán }\end{array}$ & $\begin{array}{l}\text { Suiza, Japón, } \\
\text { Turquía, Ucrania, } \\
\text { República de } \\
\text { Moldova, Islan- } \\
\text { dia, Grecia }\end{array}$ & $\begin{array}{l}\text { Cuba, Reino Uni- } \\
\text { do, Irlanda, Italia, } \\
\text { Georgia }\end{array}$ & $\begin{array}{c}\text { Kyrgyzstan, Suecia, } \\
\text { China }\end{array}$ & $\begin{array}{l}\text { Turkmenistán, Gha- } \\
\text { na, Israel, Vietnam, } \\
\text { Perú, Colombia, } \\
\text { México, Bahréin, } \\
\text { Omán, Australia, } \\
\text { Chile, Costa Rica, } \\
\text { Estados Unidos, } \\
\text { Filipinas, Nueva } \\
\text { Zelandia, República } \\
\text { de Corea, Singapur, } \\
\text { Siria, Venezuela }\end{array}$ \\
\hline $\begin{array}{l}\text { Incremento porcen- } \\
\text { tual para registro } \\
\text { marcario con } \\
\text { afectación entre } 2 \text { y } \\
3 \text { clases }\end{array}$ & $\begin{array}{l}\text { Estonia, Belarus, } \\
\text { Noruega, Finlandia, } \\
\text { Benelux, Bonaire, } \\
\text { San Marino, Bulga- } \\
\text { ria, Unión Europea, } \\
\text { Curazao, Dinamarca }\end{array}$ & $\begin{array}{l}\text { Tayikistán, Armenia, } \\
\text { Uzbequistán, Suiza, } \\
\text { Japón, Turquía, } \\
\text { Ucrania, Repúbli- } \\
\text { ca de Moldova, } \\
\text { Islandia, Grecia, } \\
\text { Cuba, Reino Unido, } \\
\text { Irlanda, Italia }\end{array}$ & $\begin{array}{c}\text { Georgia, Kyr- } \\
\text { gyzstan, Suecia, } \\
\text { China, Turkme- } \\
\text { nistán, Ghana, } \\
\text { Israel, Vietnam, } \\
\text { Perú }\end{array}$ & $\begin{array}{l}\text { Colombia, México, } \\
\text { Bahréin, Omán, } \\
\text { Australia, Chile, } \\
\text { Costa Rica, Estados } \\
\text { Unidos, Filipinas, } \\
\text { Nueva Zelandia, } \\
\text { República de Corea, } \\
\text { Singapur, Siria, } \\
\text { Venezuela }\end{array}$ & & \\
\hline
\end{tabular}

I corresponde a la tarifa inicial por registrar una (1) clase en una solicitud única de registro de marca.

$s$ es el monto de descuento de referencia. Es el número $\sum_{i=0}^{n-1} i$ de veces a aplicar el monto de descuento de referencia.

La llustración 1 muestra que a medida que aumenta el número de clases de productos y/o servicios registrados en una única solicitud de registro de marca, el descuento aplicado a la tarifa total que se cobra al titular de la marca es creciente. Esto denota la bondad del modelo propuesto en la ecuación (1), ya que al ser el descuento creciente se crean incentivos que incrementan el número de solicitudes de registros marcarios multi-clase.

La formulación de la tarifa lineal que se debe cobrar al registrar clases en una solicitud única de registro se establece de forma iterativa, comenzando por la tarifa total que se aplica desde la clase número 1 hasta clases $n$ subsecuentes, empleando para dicho

4 Fuente: elaboración propia con base en estadísticas de la OMPI (2012) y Oficinas de Propiedad Industrial para países de América Latina. 
Tabla 2. Registro de marcas $(2011)^{5}$

\begin{tabular}{|c|c|c|c|c|}
\hline Office & Residente & No-Residente & Total & $\begin{array}{c}\text { Ranking 1 } \\
\text { clase }\end{array}$ \\
\hline China & 926.330 & 107.241 & 1.033 .571 & 29 \\
Estados Unidos & 179.604 & 69.430 & 249.034 & 23 \\
Italia & 124.029 & 13.958 & 137.987 & 45 \\
República of Corea & 64.844 & 37.303 & 102.147 & 31 \\
Reino Unido & 59.906 & 15.898 & 75.804 & 27 \\
Benelux & 51.556 & 11.304 & 62.860 & 35 \\
México & 45.957 & 22.277 & 68.234 & 43 \\
Australia & 42.526 & 35.657 & 78.183 & 9 \\
Suiza & 31.372 & 48.279 & 79.651 & 14 \\
Vietnam & 23.887 & 19.349 & 43.236 & 48 \\
Ucrania & 22.429 & 29.612 & 52.041 & 6 \\
Colombia & 13.083 & 9.055 & 22.138 & 10 \\
Venezuela & 6.455 & 5.551 & 12.006 & 21 \\
Uruguay & 3.611 & 4.604 & 8.215 & 38 \\
República de & 2.142 & 9.244 & 11.386 & 20 \\
Moldova & 1.597 & 7.878 & 9.475 & 33 \\
Armenia & 326 & 6.142 & 6.468 & 7 \\
Tayikistán & 200 & 6.686 & 6.886 & 16 \\
Kyrgyzstan & 200 \\
\hline
\end{tabular}

Ilustración 1. Factor de descuento por número de cla$\operatorname{ses}^{6}$

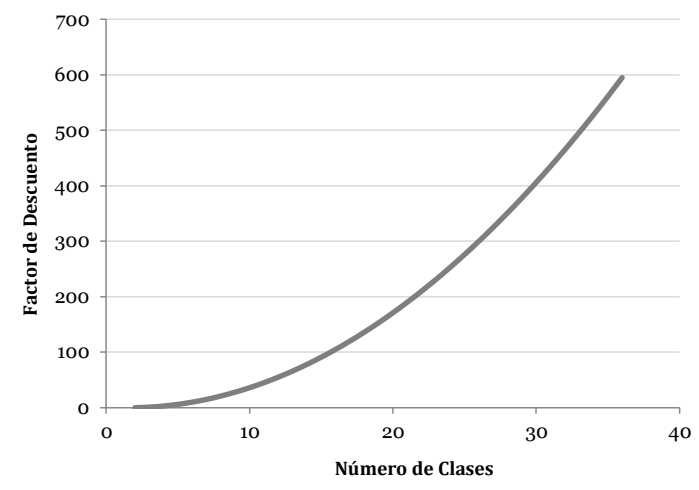

ejercicio una tarifa media inicial $\bar{p}$ y un incremento máximo de $\delta$ sobre dicha tarifa, como se muestra a continuación:

$$
\begin{gathered}
I_{1}=\bar{p}+\delta \\
I_{2}=2 I_{1}-s \\
I_{3}=3 I_{1}-3 s
\end{gathered}
$$

Por lo tanto, la tarifa lineal puede expresarse, para cada valor de $n$, de acuerdo con la siguiente expresión:

$$
I_{n}=n I-s \sum_{i=0}^{n-1} i
$$

\footnotetext{
Fuente: elaboración propia con base en estadísticas de la OMPI (2012) y Oficinas de Propiedad Industrial para países de América Latina.

6 Para una revisión sobre los fundamentos asociados a la discriminación de precios de segundo grado, véase el capítulo 14 de Nicholson (2012).
} 
Tabla 3. Solicitudes de registro de marcas $(2011)^{6}$

\begin{tabular}{|c|c|c|c|c|}
\hline Office & Residente & No-Residente & Total & $\begin{array}{c}\text { Ranking 1 } \\
\text { clase }\end{array}$ \\
\hline China & 1.273 .827 & 144.424 & 1.418 .251 & 29 \\
Estados Unidos & 319.311 & 92.703 & 412.014 & 23 \\
Turquía & 152.261 & 32.678 & 184.939 & 30 \\
Japón & 142.157 & 47.060 & 189.217 & 4 \\
República de Corea & 132.864 & 41.433 & 174.297 & 31 \\
Italia & 85.129 & 12.925 & 98.054 & 45 \\
Reino Unido & 72.109 & 17.131 & 89.240 & 27 \\
México & 71.091 & 29.190 & 100.281 & 43 \\
Australia & 69.058 & 43.577 & 112.635 & 9 \\
Benelux & 60.081 & 15.711 & 75.792 & 35 \\
Vietnam & 34.718 & 21.420 & 56.138 & 48 \\
Suiza & 34.264 & 50.747 & 85.011 & 14 \\
Colombia & 16.976 & 12.108 & 29.084 & 10 \\
Venezuela & 11.066 & 8.521 & 19.587 & 21 \\
Uzbekistán & 4.500 & 7.608 & 12.108 & 2 \\
Cuba & 600 & 4.132 & 4.732 & 13 \\
Kyrgyzstan & 271 & 7.117 & 7.388 & 16 \\
Bahréin & 269 & 10.599 & 10.868 & 24 \\
\hline
\end{tabular}

Reemplazando $I_{n}=n \cdot p$ en la tarifa lineal se obtiene que:

$$
\frac{n \cdot \delta}{s}=\sum_{i=0}^{n-1} i
$$

De otro lado se sabe que:

$$
\sum_{i=0}^{n-1} i=n^{2}-\sum_{i=1}^{n} i
$$

De esta manera, es posible mostrar que:

$$
\sum_{i=1}^{n} i=\frac{n^{2}+n}{2}
$$

Finalmente, reemplazando en las tres últimas ecuaciones se obtiene la ecuación 6 :

$$
n^{*}=\frac{2 \delta}{s}+1
$$

Esta última expresión representa el número óptimo de clases a registrar en una solicitud única de marca $\left(n^{*}\right)$ que permite en el contexto de un sistema de registro multi-clase dispuesto por el TLT, que el ingreso generado por la tabla propuesta de tarifas diferenciadas, sea igual a aquel que produciría en el sistema de registro con tarifa única.

\subsection{Aplicación al caso concreto del Sistema Marcario Multi-clase en Colombia}

A partir del comportamiento histórico del registro de clase multi-clase en Colombia, es posible incorporar

6 Fuente: elaboración propia con base en estadísticas de la OMPI (2012). 
algunos puntos que dan una idea preliminar de la demanda existente en Colombia acerca del servicio en mención. De acuerdo con un estudio realizado en el año 2011, cada titular de una marca posee, en promedio, derechos en tres clases de productos o servicios (Vargas, 2012). Dicha tendencia se sustenta con la información suministrada por la Delegatura de Propiedad Industrial de la Superintendencia de Industria y Comercio, según la cual para el año 2011, cerca de 8.622 solicitudes de registro de marca hacen referencia a entre 2 y 3 clases, lo que corresponde al $60,8 \%$ y $19,6 \%$, respectivamente. En otras palabras, cerca del $80,4 \%$ de las solicitudes de registro de marca se relacionaban con 2 ó 3 clases (Ilustración 2).

Ilustración 2. Número de clases registradas por titular de Marca $(2011)^{7}$

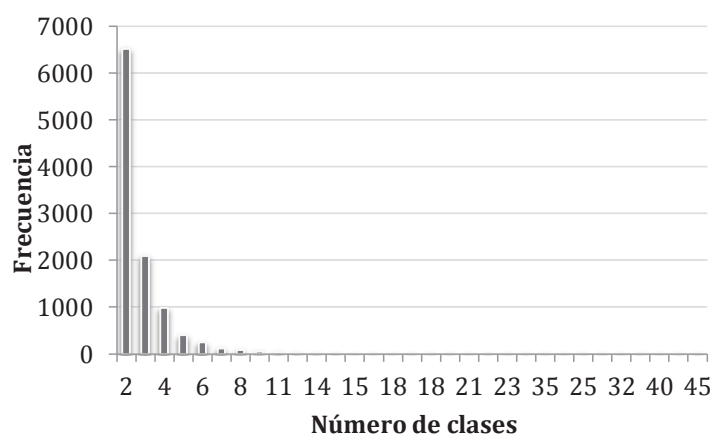

En este contexto, la aplicación de la metodología descrita en la sección inmediatamente anterior pretende encontrar la tabla óptima de tarifas diferenciadas, según el número de clases registradas en cada solicitud única de registro de marca, y el impacto que tiene su implementación sobre los ingresos de la Entidad, derivados de las actividades de Propiedad Industrial.

Para alcanzar los anteriores objetivos se propone la parametrización del modelo teórico, utilizando los siguientes insumos: $\bar{p}$ : Tarifa media inicial que se cobra por registrar una (1) clase en cada registro de marca. Hace referencia a la tasa de registro de marca del año 2011 cuya cuantía es de $\$ 728.000$.

$n$ : Número total de marcas a registrar. Hace referencia a la cantidad proyectada de registros de marcas en el año 2012 (31.000 marcas y lemas comerciales) $)^{8}$

$\Delta$ : Incremento máximo sobre la tarifa inicial. Para el ejercicio se propone que dicho valor corresponda al ajuste por inflación esperada para el 2012 (3\%).

$I_{1}$ : Tarifa total inicial de registrar solo una clase en cada registro único de marca. Hace referencia a la ecuación $I_{1}=\bar{p}+\delta$. Con los datos presentados, la cuantía de la tarifa total media inicial es de $\$ 750.000$, tasa actual que se aplica para las solicitudes de registro de marca (SIC, 2011).

Al aplicar las ecuaciones obtenidas en la sección anterior, y utilizando la parametrización anterior, se obtiene que para un número óptimo de clases a registrar en una solicitud única de registro de marca $n^{*}=3$, la estructura de tarifas diferenciadas establece que cada clase adicional tendría un descuento de $\$ 11.000$, reflejando un descuento porcentual creciente, a medida que aumenta el número de clases por solicitud única de registro de marca. Se considera un límite de 45 clases por registrar en cada solicitud única de registro de marca, al ser este el número de clases de productos y/o servicios dispuesto en la Clasificación de Niza (OMPI, 2014).

El impacto esperado del sistema multi-clase de registro de marcas dispuesto por el TLT, sobre los ingresos de la autoridad de propiedad industrial, se analiza por medio de la comparación de los ingresos que la Entidad percibiría con la aplicación de las tarifas diferenciadas, frente a los ingresos que se recibirían si al registro multi-clase de marcas se aplicara una tarifa única, que no discrimina por cantidad de cla-

Fuente: elaboración propia con base en la información suministrada por la Delegatura de Propiedad Industrial de la Superintendencia de Industria y Comercio.

8 Esta información se obtuvo del Sistema de Trámites de la SIC. 
Tabla 4. Tabla de tarifas diferenciadas ${ }^{9}$

\begin{tabular}{|c|c|c|c|}
\hline $\begin{array}{l}\text { Clases por solicitud Únicade } \\
\text { Registro de Marca (A) }\end{array}$ & Tarifa media (B) & Tarifa total $(C)=(A)^{\star}(B)$ & $\begin{array}{l}\text { Tarifa total Descuento PYME } \\
\text { (C) }-25 \%\end{array}$ \\
\hline 1 & $\$ 750.000$ & $\$ 750.000$ & $\$ 562.500$ \\
\hline 2 & $\$ 739.000$ & $\$ 1.478 .000$ & $\$ 1.108 .500$ \\
\hline 3 & $\$ 728.000$ & $\$ 2.184 .000$ & $\$ 1.638 .000$ \\
\hline 4 & $\$ 717.000$ & $\$ 2.868 .000$ & $\$ 2.151 .000$ \\
\hline 5 & $\$ 706.000$ & $\$ 3.530 .000$ & $\$ 2.647 .500$ \\
\hline 6 & $\$ 695.000$ & $\$ 4.170 .000$ & $\$ 3.127 .500$ \\
\hline 7 & $\$ 684.000$ & $\$ 4.788 .000$ & $\$ 3.591 .000$ \\
\hline 8 & $\$ 673.000$ & $\$ 5.384 .000$ & $\$ 4.038 .000$ \\
\hline 9 & $\$ 662.000$ & $\$ 5.958 .000$ & $\$ 4.468 .500$ \\
\hline 10 & $\$ 651.000$ & $\$ 6.510 .000$ & $\$ 4.882 .500$ \\
\hline 11 & $\$ 640.000$ & $\$ 7.040 .000$ & $\$ 5.280 .000$ \\
\hline 12 & $\$ 629.000$ & $\$ 7.548 .000$ & $\$ 5.661 .000$ \\
\hline 13 & $\$ 618.000$ & $\$ 8.034 .000$ & $\$ 6.025 .500$ \\
\hline 14 & $\$ 607.000$ & $\$ 8.498 .000$ & $\$ 6.373 .500$ \\
\hline 15 & $\$ 596.000$ & $\$ 8.940 .000$ & $\$ 6.705 .000$ \\
\hline 16 & $\$ 585.000$ & $\$ 9.360 .000$ & $\$ 7.020 .000$ \\
\hline 17 & $\$ 574.000$ & $\$ 9.758 .000$ & $\$ 7.318 .500$ \\
\hline 18 & $\$ 563.000$ & $\$ 10.134 .000$ & $\$ 7.600 .500$ \\
\hline 19 & $\$ 552.000$ & $\$ 10.488 .000$ & $\$ 7.866 .000$ \\
\hline 20 & $\$ 541.000$ & $\$ 10.820 .000$ & $\$ 8.115 .000$ \\
\hline 21 & $\$ 530.000$ & $\$ 11.130 .000$ & $\$ 8.347 .500$ \\
\hline 22 & $\$ 519.000$ & $\$ 11.418 .000$ & $\$ 8.563 .500$ \\
\hline 23 & $\$ 508.000$ & $\$ 11.684 .000$ & $\$ 8.763 .000$ \\
\hline 24 & $\$ 497.000$ & $\$ 11.928 .000$ & $\$ 8.946 .000$ \\
\hline 25 & $\$ 486.000$ & $\$ 12.150 .000$ & $\$ 9.112 .500$ \\
\hline 26 & $\$ 475.000$ & $\$ 12.350 .000$ & $\$ 9.262 .500$ \\
\hline 27 & $\$ 464.000$ & $\$ 12.528 .000$ & $\$ 9.396 .000$ \\
\hline 28 & $\$ 453.000$ & $\$ 12.684 .000$ & $\$ 9.513 .000$ \\
\hline 29 & $\$ 442.000$ & $\$ 12.818 .000$ & $\$ 9.613 .500$ \\
\hline 30 & $\$ 431.000$ & $\$ 12.930 .000$ & $\$ 9.697 .500$ \\
\hline 31 & $\$ 420.000$ & $\$ 13.020 .000$ & $\$ 9.765 .000$ \\
\hline 32 & $\$ 409.000$ & $\$ 13.088 .000$ & $\$ 9.816 .000$ \\
\hline 33 & $\$ 398.000$ & $\$ 13.134 .000$ & $\$ 9.850 .500$ \\
\hline 34 & $\$ 387.000$ & $\$ 13.158 .000$ & $\$ 9.868 .500$ \\
\hline
\end{tabular}

9 Fuente: elaboración propia con base en la información suministrada por la Delegatura de Propiedad Industrial de la Superintendencia de Industria y Comercio. 


\begin{tabular}{|c|c|c|c|}
\hline $\begin{array}{c}\text { Clases por solicitud Únicade } \\
\text { Registro de Marca (A) }\end{array}$ & Tarifa media $(\mathbf{B})$ & Tarifa total $(\mathbf{C})=(\mathbf{A})^{*}(\mathbf{B})$ & $\begin{array}{c}\text { Tarifa total Descuento PYME } \\
\mathbf{( C )}-\mathbf{2 5} \%\end{array}$ \\
\hline 35 & $\$ 376.000$ & $\$ 13.160 .000$ & $\$ 9.870 .000$ \\
36 & $\$ 365.000$ & $\$ 13.140 .000$ & $\$ 9.855 .000$ \\
37 & $\$ 354.000$ & $\$ 13.098 .000$ & $\$ 9.823 .500$ \\
38 & $\$ 343.000$ & $\$ 13.034 .000$ & $\$ 9.775 .500$ \\
39 & $\$ 332.000$ & $\$ 12.948 .000$ & $\$ 9.711 .000$ \\
40 & $\$ 321.000$ & $\$ 12.840 .000$ & $\$ 9.630 .000$ \\
41 & $\$ 310.000$ & $\$ 12.710 .000$ & $\$ 9.532 .500$ \\
42 & $\$ 299.000$ & $\$ 12.558 .000$ & $\$ 9.418 .500$ \\
43 & $\$ 288.000$ & $\$ 12.384 .000$ & $\$ 9.288 .000$ \\
44 & $\$ 277.000$ & $\$ 12.188 .000$ & $\$ 9.141 .000$ \\
45 & $\$ 266.000$ & $\$ 11.970 .000$ & $\$ 8.977 .500$ \\
\hline
\end{tabular}

ses registradas en cada solicitud única de registro de marca.

Para tal efecto, se aplicó la frecuencia del número de clases registradas por los titulares de marca durante el año 2011 sobre las 31.000 solicitudes de registro de marca que se esperaron para el año 2012. A partir de esta distribución, se proyectaron los ingresos por tal concepto, bajo el esquema de tarifas diferenciadas frente a los derivados de la implementación de la tarifa actual única de $\$ 750.000$.

Como se observa en el Tabla 4 (y la Tabla A1 del Anexo), de las 31.000 solicitudes de registro de marca previstas para el año 2012, aproximadamente 18.844 y 6.075 solicitudes hacían referencia a la solicitud única de registro de marca de 2 y 3 clases, respectivamente. Dada esta distribución en el número de clases en cada solicitud, la implementación de una estructura de tarifas diferenciadas permitiría percibir ingresos de $\$ 22.870 .277 .457$ frente a $\$ 23.178 .468 .208$ derivados de la aplicación de una tarifa única de $\$ 750.000$. Esto representa una caída del 1,5\%, cerca de $\$ 308.190 .751$ en los ingresos de la Entidad obtenidos de las solicitudes de registro de marcas, lo que demuestra que el sistema de tarifas diferenciadas corresponde a un esquema que amortigua el descenso de los ingresos de la Entidad, dada la entrada en vigor del Artículo 6 del TLT.

\section{Consideraciones Finales}

Este documento ha presentado el modelo empleado para la determinación de tarifas marcarias multi-clase en 2012, a propósito de la adhesión de Colombia al TLT, a partir de los datos suministrados por la Delegatura de Propiedad Industrial de la Superintendencia de Industria y Comercio. Lo anterior en atención a que dicho tratado tiene por objeto facilitar el sistema de registro de marcas, a través de la simplificación y la armonización de los procedimientos y la eliminación de obstáculos, de forma tal que las solicitudes de registro de marcas y la administración de los mismos resulten uniformes en todos los países miembros del Tratado.

Dentro de los hallazgos más importantes del estudio fue posible identificar que las tarifas aplicadas para registro de marcas en Colombia, si bien no eran las más altas en el mundo, ameritaban una revisión. Un ejercicio que hiciera esta comparación no había sido elaborado para el caso colombiano. Igualmente, en análisis de los hechos estilizados permitió establecer que no hay una relación inversa inequívoca entre la tarifa marcaria y el número de solicitudes, lo que da espacio a considerar múltiples factores que pueden estar incidiendo en la demanda del servicio de registros marcarios, distintos a su precio. En cuanto al aporte realizado a la literatura, este documento corresponde 
al primer trabajo que aplica una tarifa en dos tramos a los costos de registro de propiedad industrial.

Reconociendo cierta limitación de información en cuanto a la demanda del servicio, ya que se tomaron datos históricos del año inmediatamente anterior (2011) al período en el que se propuso el ejercicio, se planteó un sistema de tarifas diferenciadas, siguiendo la teoría de la discriminación de precios de segundo grado, según la cual se establece un descuento escalonado de acuerdo con la cantidad de clases que se registren en una única solicitud de registro de marca. Se encontró que este sistema de tarifas diferenciadas amortigua el descenso de los ingresos de la autoridad administradora de propiedad industrial en Colombia, proyectando una caída del 1,5\% sobre los ingresos derivados de este concepto, ante una plena inelasticidad de la demanda al cambio en el precio en el corto plazo.

Queda propuesto como agenda futura de investigación en este campo, avanzar más detalladamente en una caracterización de la demanda de servicios de propiedad industrial en Colombia y evaluar el impacto ex post de la puesta en marcha de este sistema de tarifas.

\section{Referencias}

Bonnet, C. \& Dubois, P. (2010). "Inference on vertical contracts between manufacturers and retailers allowing for nonlinear pricing and resale price maintenance". En: The RAND Journal of Economics, 41(1): 139-164.

Braverman, A., Guasch, J. \& Salop, S. (1983). "Defects in Disneyland: Quality Control as a Two-Part Tariff". En: The Review of Economic Studies, 50(1): 121-131.

Chang, R-Y., Hwang, H. \& Peng, C-H. (2013). "Technology licensing, R\&D and welfare”. En: Economics Letters, 118 (2): 396-399.

Chen, J., Zhang, H. \& Sun, Y. (2012). "Implementing coordination contracts in a manufacturer Stackelberg dual-channel supply chain". En: Omega, 40(5): 571-583.

Corcione, M. (2010). "El TLT (Trade Law Treaty) y los retos para la Superintendencia de Industria y Comercio como oficina de propiedad industrial". En: Revista La Propiedad Material, 14: 319-326.

Cornia, M., Gerardi, K. \& Shapiro, A. (2011). Price Discrimination and Business-Cycle Risk (Working Paper 2011-3). Atlanta, Federal Reserve Bank Of Atlanta.

Felder, S. (2004). "Drug price regulation under consumer moral hazard Two-part tariffs, uniform price or third-degree price discrimination?". En: The European Journal of Health Economics, 5(4): 324-329.
Ferreira, F. \& Bode, O. R. (2013). "Licensing endogenous costreduction in a differentiated Stackelberg model". En: Communications in Nonlinear Science and Numerical Simulation, 18(2): 308-315.

Gotlibovski, C. \& Kahana, N. (2009). "Second-Degree Price Discrimination: A Graphical and Mathematical Approach". En: Journal of Economic Education, 40(1): 68-79.

Inderst, R. \& Shaffer, G. (2009). "Market power, price discrimination, and allocative efficiency in intermediate-goods markets". En: The RAND Journal of Economics, 40(4): 658-672.

Kim, S.L., Park, C.H. \& Lee, S.H. (2012). "Environmental Tax and Licensing a Patent for Clean Technology Management". En: Procedia - Social and Behavioral Sciences, 57(9): 95-101.

Lambertini, L. (2013). "Coordinating static and dynamic supply chains with advertising through two-part tariffs". Automatica, Doi: http://dx.doi.org/10.1016/j.automatica.2013.11.024

Li, Y. \& Yanagawa, T. (2011). "Patent licensing of Stackelberg manufacturer in a differentiated product market". En: International Journal of Economic Theory, 7(1): 7-20.

Ma, P, Wang, H. \& Shang, J. (2013). "Contract design for two-stage supply chain coordination: Integrating manufacturer-quality and retailer-marketing efforts". En: International Journal of Production Economics, 146 (2): 745-755.

Mendonca, S., Pereira, T. \& Godinho, M. (2004). Trademarks as an Indicator of Innovation and Industrial Change. En: Research Policy, 33: 1385-1404.

Matsui, K. (2012). "Strategic upfront marketing channel integration as an entry barrier". En: European Journal of Operational Research, 220 (3): 865-875.

Mukherjee, A. \& Mukherjee, S. (2013). "Technology licensing and innovation". En: Economics Letters, 120 (3): 499-502.

Nicholson, W. (2012). Microeconomic Theory: Basic Principles and Extensions. Ed South Western. Cengage Learning.

Oliveira, F., Ruiz, C. \& Conejo, A. (2013). "Contract design and supply chain coordination in the electricity industry". En: European Journal of Operational Research, 227 (3): 527-537.

OMPI (1994). Tratado sobre Derecho de Marcas. Disponible en: http://www.wipo.int/treaties/es/ip/ttl/trtdocs_wo027.html

OMPI (2012). Individual Fees under the Madrid Protocol. Disponible en: http://www.wipo.int/madrid/en/madridgazette/remarks/ ind taxes.html

OMPI (2014). List of Goods and Services by Class Order. Disponible en: http://web2.wipo.int/nicepub/edition-20140101/tax onomy $/ \#$ ?pagination $=$ no\&lang $=e n \&$ mode $=$ flat\&explanato ry_notes $=$ hide\&basic_numbers $=$ show \&_suid $=1389630084$ 7410142359155526394

Rosellon, J., Tregear, J. \& Zenon, E. (2012). El modelo HRV para expansión óptima de redes de transmisión: una aplicación a la red eléctrica de Ontario. En: Economía Mexicana, Nueva Época, 21(1): 133-173

Ruiz, E. \& Rosellón, J. (2012). "Transmission investment in the Peruvian electricity market: Theory and applications". En: Energy Policy, 47: 238-245.

Steele Santos, P., Coradi Leme, R. \& Galvão, L. (2012). "On the electrical two-part tariff-The Brazilian perspective". En: Energy Policy, 40: 123-130.

Superintendencia de Industria y Comercio (2012). Presupuesto de la Superintendencia de Industria y Comercio ante el Ministerio de Hacienda y Crédito Público. Disponible en http://www.sic.gov. 


\section{DISCRIMINACIÓN DE PRECIOS DE SEGUNDO GRADO: EL CASO DE TARIFAS}

MULTI-CLASE PARA EL REGISTRO MARCARIO EN COLOMBIA

co/recursos user/documentos/Conozcanos/Gestion/Presupuesto/ Formularios Anteproyecto 2012.pdf

Superintendencia de Industria y Comercio SIC (2011). Resolución número 75.858 de 2011 "Solicitud de registro de marcas de productos o servicios, de marcas colectivas, de marcas de certificación y de lemas comerciales". Bogotá: Superintendencia de Industria y Comercio.

Superintendencia de Industria y Comercio - SIC (2008). Marcas. Guía de propiedad industrial. Bogotá: SIC. Disponible en: http:// www.sic.gov.co/recursos_user/documentos/propiedad_industrial/ WEB/assets/pdf/Guia Marcas.pdf

Vargas, C. (2012). Estudio Tasas en Propiedad Industrial. Bogotá: Superintendencia de Industria y Comercio.
Wang, X. H. (2002). "Fee versus royalty licensing in a differentiated Cournot duopoly". En: Journal of Economics and Business, 54: 253-66.

Watika, S. (2001). "Why has the Unemployment Rate Been so Low in Japan? An Explanation by Two-Part Wage Bargaining". En: Japanese Economic Review, 52: 116-133.

Willington, M. \& Li Ning, J. (2014). "Regulating a monopoly with universal service obligations: The role of flexible tariffschemes. En: Telecommunications Policy, 38(1): 32-48.

Yao, S. (2002). "Privilege and Corruption The Problems of China's Socialist Market Economy". En: American Journal of Economics and Sociology, 61(1): 279-299. 


\section{Anexos}

Ilustración A1. Valor de registro de una marca con afectación a una sola clase (cifras en francos suizos - 4 de diciembre de 2012) ${ }^{10}$

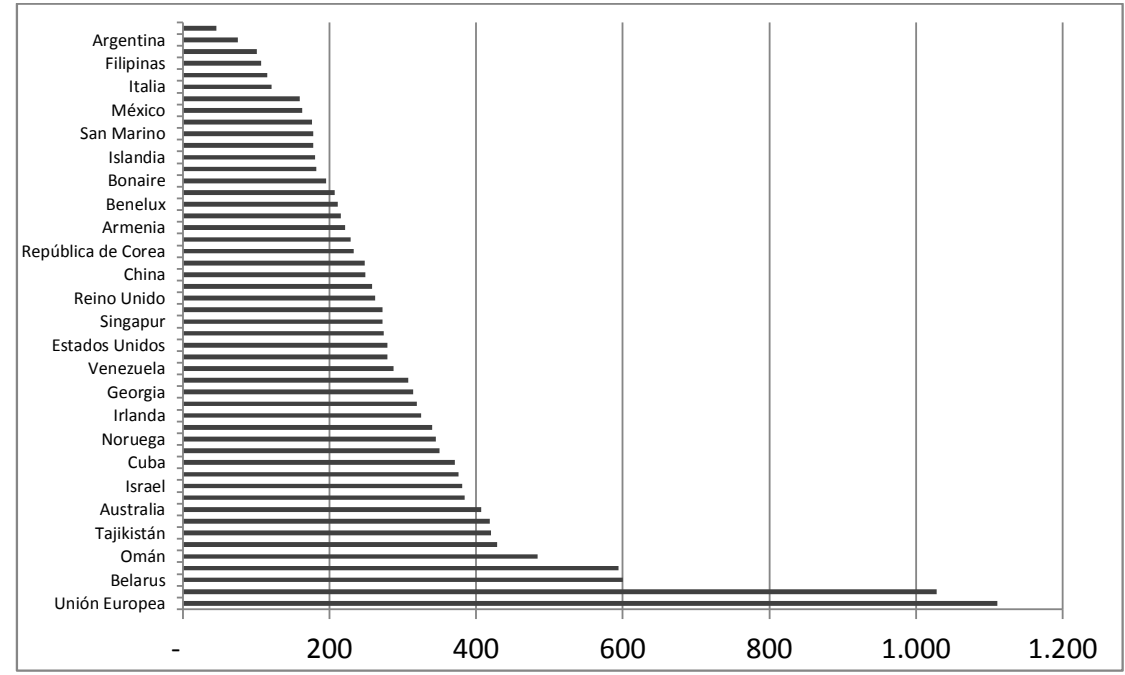

Nota. El valor de registro marcario para Uruguay oscila entre 130 y 182 francos suizos dependiendo si la marca es emblemática o denominativa.

Ilustración A2. Valor de registro de una marca con afectación a dos clases (cifras en francos suizos - 4 de diciembre de 2012) ${ }^{11}$

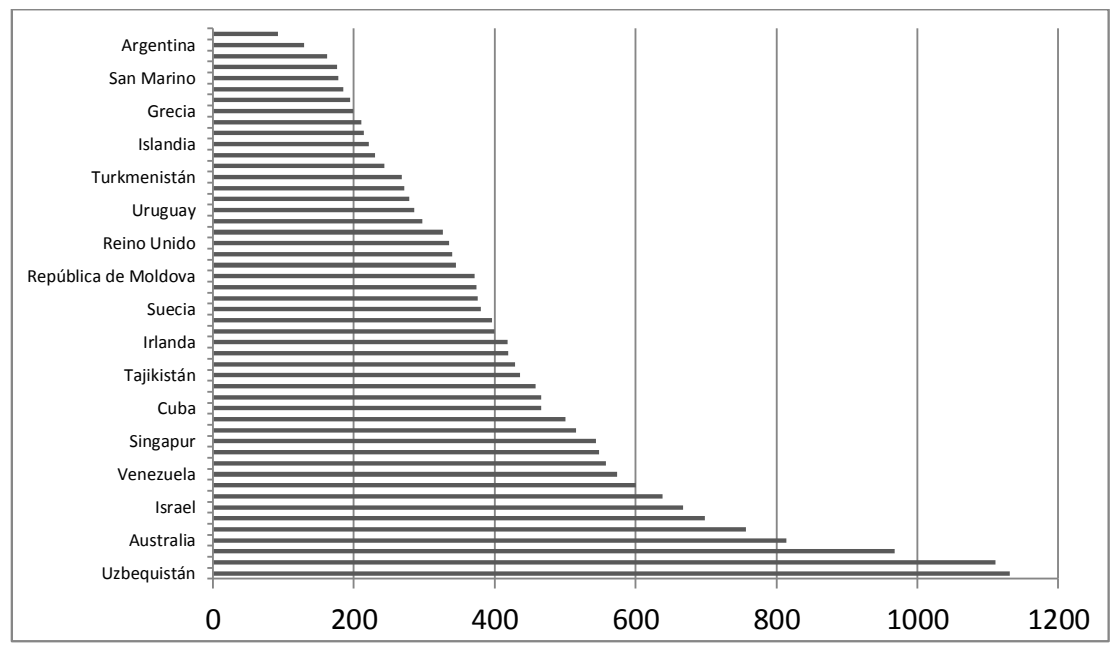

Nota. El valor de registro marcario para Uruguay oscila entre 208 y 286 francos suizos y Argentina entre 93 y 129 francos suizos.

10 Fuente: elaboración propia con base en estadísticas de WIPO y Oficinas de Propiedad Industrial para países de América Latina.

11 Fuente: elaboración propia a partir de estadísticas de WIPO y Oficinas de Propiedad Industrial para países de América Latina. 
Ilustración A3. Valor de registro de una marca con afectación a tres clases (cifras en francos suizos - 4 de diciembre de 2012) $)^{12}$

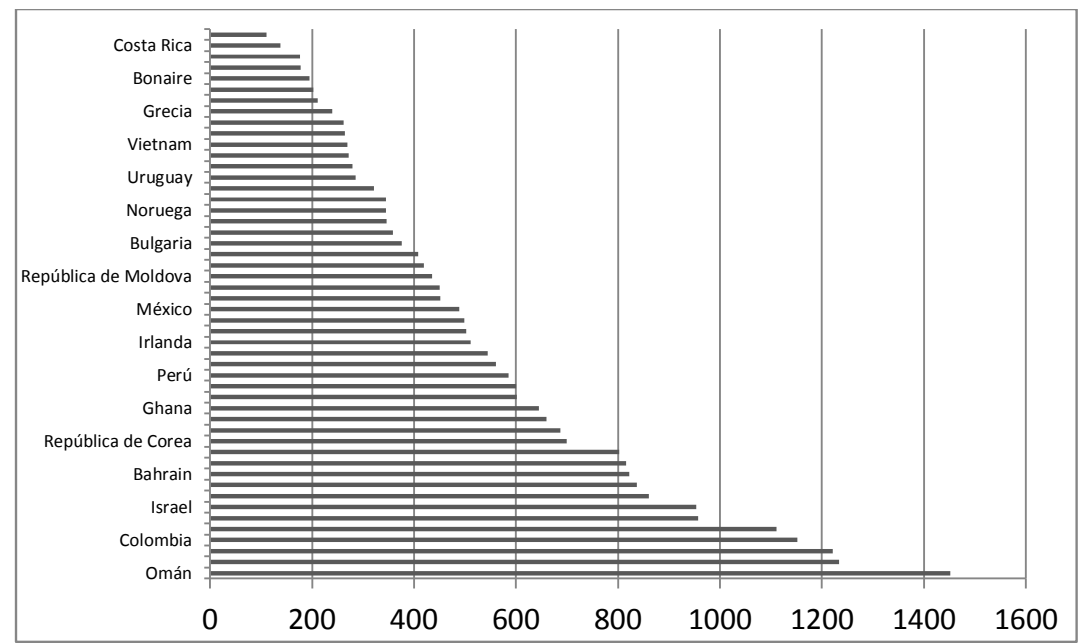

Nota. El valor de registro marcario para Uruguay oscila entre 286 y 338 francos suizos y Argentina entre 111 y 183 francos suizos.

Ilustración A4. Incremento en el valor del registro para marca colectiva ${ }^{13}$

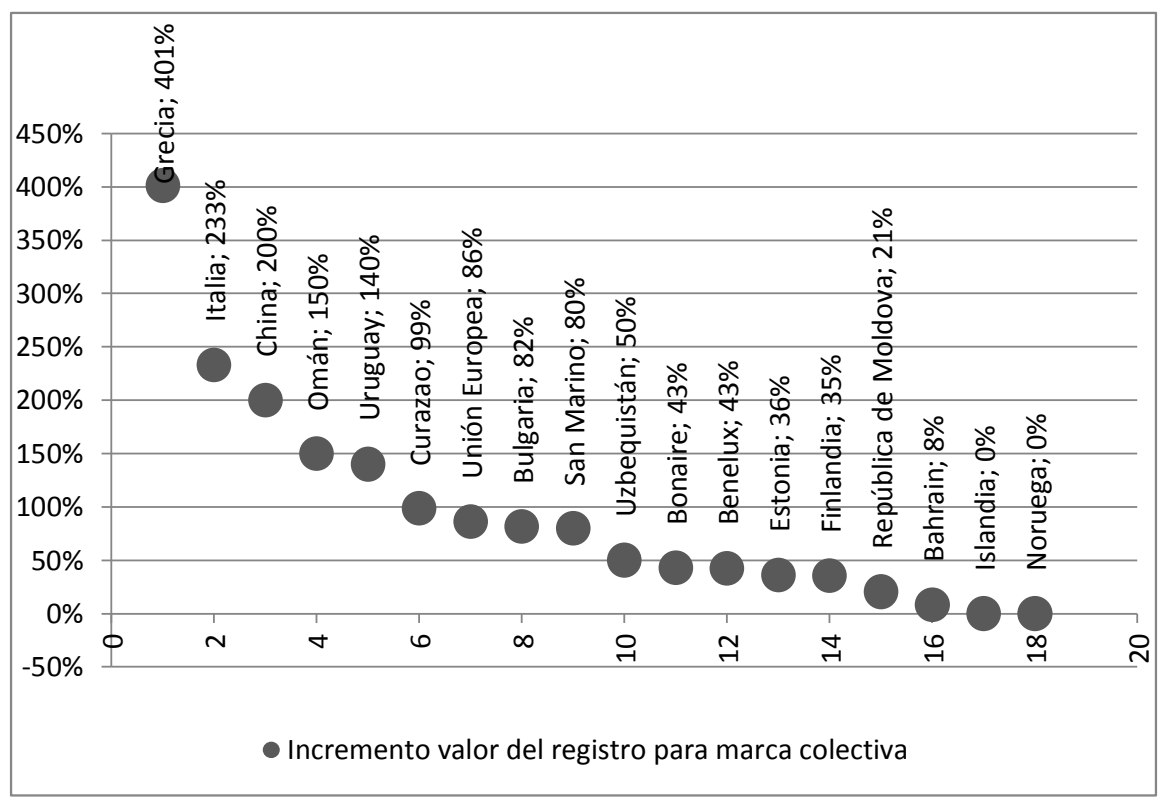

12 Fuente: elaboración propia con base en estadísticas de WIPO y Oficinas de Propiedad Industrial para países de América Latina. 13 Fuente: elaboración propia con base en estadísticas de WIPO y Oficinas de Propiedad Industrial para países de América Latina. 
Tabla A1. Proyección del impacto sobre los ingresos de la entidad (2012) ${ }^{14}$

\begin{tabular}{|c|c|c|c|c|c|c|c|c|}
\hline \multirow{3}{*}{$\begin{array}{c}\text { Número } \\
\text { Total de } \\
\text { Solicitudes } \\
\text { Clase }\end{array}$} & \multirow{3}{*}{$\begin{array}{c}31.000 \\
\text { Número de } \\
\text { Clases sobre } \\
\text { el Total de } \\
\text { Solicitudes (\%) }\end{array}$} & & & & & & & \\
\hline & & & \multicolumn{2}{|c|}{$\begin{array}{l}\text { Solicitud con Tarifa } \\
\text { Diferenciada }\end{array}$} & \multicolumn{2}{|c|}{ Solicitud con Tarifa Única } & \multirow[b]{2}{*}{$\begin{array}{c}\text { Diferencia (A) } \\
\text { - (B) }\end{array}$} & \multirow[b]{2}{*}{$\begin{array}{l}\text { Varia- } \\
\text { ción \% }\end{array}$} \\
\hline & & $\begin{array}{c}\text { Total de } \\
\text { Solicitu- } \\
\text { des }\end{array}$ & $\begin{array}{l}\text { Ingreso } \\
\text { Medio }\end{array}$ & $\begin{array}{c}\text { Ingreso } \\
\text { Proyectado (A) }\end{array}$ & $\begin{array}{l}\text { Ingreso } \\
\text { Medio }\end{array}$ & $\begin{array}{c}\text { Ingreso } \\
\text { Proyectado (B) }\end{array}$ & & \\
\hline 2 & $60,79 \%$ & 18844 & $\$ 750.000$ & $\$ 14.132 .947 .977$ & $\$ 750.000$ & $\$ 14.132 .947 .977$ & $\$ 0$ & $0,0 \%$ \\
\hline 3 & $19,60 \%$ & 6075 & $\$ 739.000$ & $\$ 4.489 .531 .792$ & $\$ 750.000$ & $\$ 4.556 .358 .382$ & $-\$ 66.826 .590$ & $-1,5 \%$ \\
\hline 4 & $9,24 \%$ & 2864 & $\$ 728.000$ & \$2.085.109.827 & $\$ 750.000$ & $\$ 2.148 .121 .387$ & $-\$ 63.011 .561$ & $-2,9 \%$ \\
\hline 5 & $3,79 \%$ & 1173 & $\$ 717.000$ & $\$ 841.335 .260$ & $\$ 750.000$ & $\$ 880.057 .803$ & $-\$ 38.722 .543$ & $-4,4 \%$ \\
\hline 6 & $2,33 \%$ & 723 & $\$ 706.000$ & $\$ 510.115 .607$ & $\$ 750.000$ & $\$ 541.907 .514$ & $-\$ 31.791 .908$ & $-5,9 \%$ \\
\hline 7 & $1,16 \%$ & 358 & $\$ 695.000$ & $\$ 249.075 .145$ & $\$ 750.000$ & $\$ 268.786 .127$ & $-\$ 19.710 .983$ & $-7,3 \%$ \\
\hline 8 & $0,86 \%$ & 266 & $\$ 684.000$ & $\$ 181.872 .832$ & $\$ 750.000$ & $\$ 199.421 .965$ & $-\$ 17.549 .133$ & $-8,8 \%$ \\
\hline 9 & $0,53 \%$ & 165 & $\$ 673.000$ & $\$ 110.869 .942$ & $\$ 750.000$ & $\$ 123.554 .913$ & $-\$ 12.684 .971$ & $-10,3 \%$ \\
\hline 10 & $0,39 \%$ & 121 & $\$ 662.000$ & $\$ 80.358 .382$ & $\$ 750.000$ & $\$ 91.040 .462$ & $-\$ 10.682 .081$ & $-11,7 \%$ \\
\hline 11 & $0,26 \%$ & 81 & $\$ 651.000$ & $\$ 52.682 .081$ & $\$ 750.000$ & $\$ 60.693 .642$ & $-\$ 8.011 .561$ & $-13,2 \%$ \\
\hline 12 & $0,21 \%$ & 66 & $\$ 640.000$ & $\$ 42.543 .353$ & $\$ 750.000$ & $\$ 49.855 .491$ & $-\$ 7.312 .139$ & $-14,7 \%$ \\
\hline 13 & $0,10 \%$ & 32 & $\$ 618.000$ & $\$ 19.647 .399$ & $\$ 750.000$ & $\$ 23.843 .931$ & $-\$ 4.196 .532$ & $-17,6 \%$ \\
\hline 14 & $0,07 \%$ & 20 & $\$ 596.000$ & $\$ 12.057 .803$ & $\$ 750.000$ & $\$ 15.173 .410$ & $-\$ 3.115 .607$ & $-20,5 \%$ \\
\hline 15 & $0,06 \%$ & 17 & $\$ 585.000$ & $\$ 10.144 .509$ & $\$ 750.000$ & $\$ 13.005 .780$ & $-\$ 2.861 .272$ & $-22,0 \%$ \\
\hline 16 & $0,06 \%$ & 17 & $\$ 574.000$ & $\$ 9.953 .757$ & $\$ 750.000$ & $\$ 13.005 .780$ & $-\$ 3.052 .023$ & $-23,5 \%$ \\
\hline 17 & $0,06 \%$ & 17 & $\$ 563.000$ & $\$ 9.763 .006$ & $\$ 750.000$ & $\$ 13.005 .780$ & $-\$ 3.242 .775$ & $-24,9 \%$ \\
\hline 18 & $0,05 \%$ & 14 & $\$ 552.000$ & $\$ 7.976 .879$ & $\$ 750.000$ & $\$ 10.838 .150$ & $-\$ 2.861 .272$ & $-26,4 \%$ \\
\hline 19 & $0,03 \%$ & 9 & $\$ 541.000$ & $\$ 4.690 .751$ & $\$ 750.000$ & $\$ 6.502 .890$ & $-\$ 1.812 .139$ & $-27,9 \%$ \\
\hline 20 & $0,02 \%$ & 6 & $\$ 530.000$ & $\$ 3.063 .584$ & $\$ 750.000$ & $\$ 4.335 .260$ & $-\$ 1.271 .676$ & $-29,3 \%$ \\
\hline 21 & $0,02 \%$ & 6 & $\$ 519.000$ & $\$ 3.000 .000$ & $\$ 750.000$ & $\$ 4.335 .260$ & $-\$ 1.335 .260$ & $-30,8 \%$ \\
\hline 22 & $0,02 \%$ & 6 & $\$ 508.000$ & $\$ 2.936 .416$ & $\$ 750.000$ & $\$ 4.335 .260$ & $-\$ 1.398 .844$ & $-32,3 \%$ \\
\hline 23 & $0,01 \%$ & 3 & $\$ 497.000$ & $\$ 1.436 .416$ & $\$ 750.000$ & $\$ 2.167 .630$ & $-\$ 731.214$ & $-33,7 \%$ \\
\hline 24 & $0,01 \%$ & 3 & $\$ 486.000$ & $\$ 1.404 .624$ & $\$ 750.000$ & $\$ 2.167 .630$ & $-\$ 763.006$ & $-35,2 \%$ \\
\hline 25 & $0,01 \%$ & 3 & $\$ 475.000$ & $\$ 1.372 .832$ & $\$ 750.000$ & $\$ 2.167 .630$ & $-\$ 794.798$ & $-36,7 \%$ \\
\hline 26 & $0,01 \%$ & 3 & $\$ 464.000$ & $\$ 1.341 .040$ & $\$ 750.000$ & $\$ 2.167 .630$ & $-\$ 826.590$ & $-38,1 \%$ \\
\hline 27 & $0,01 \%$ & 3 & $\$ 453.000$ & $\$ 1.309 .249$ & $\$ 750.000$ & $\$ 2.167 .630$ & $-\$ 858.382$ & $-39,6 \%$ \\
\hline 28 & $0,01 \%$ & 3 & $\$ 442.000$ & $\$ 1.277 .457$ & $\$ 750.000$ & $\$ 2.167 .630$ & $-\$ 890.173$ & $-41,1 \%$ \\
\hline 29 & $0,01 \%$ & 3 & $\$ 431.000$ & $\$ 1.245 .665$ & $\$ 750.000$ & $\$ 2.167 .630$ & $-\$ 921.965$ & $-42,5 \%$ \\
\hline 30 & $0,01 \%$ & 3 & $\$ 420.000$ & $\$ 1.213 .873$ & $\$ 750.000$ & $\$ 2.167 .630$ & $-\$ 953.757$ & $-44,0 \%$ \\
\hline Total Ingresos & & & & \$22.870.277.457 & & $\$ 23.178 .468 .208$ & $-\$ 308.190 .751$ & $-1,5 \%$ \\
\hline
\end{tabular}

14 Fuente: elaboración propia con base en la información suministrada por la Delegatura de Propiedad Industrial de la Superintendencia de Industria y Comercio, 2012. 